Case Report

\title{
IgG4-Related Kidney Disease: Report of a Case Presenting as a Renal Mass
}

\author{
Daniele Bianchi, ${ }^{1}$ Luca Topazio, ${ }^{1}$ Gabriele Gaziev, ${ }^{1}$ Valerio Iacovelli, ${ }^{1}$ Pierluigi Bove, \\ Alessandro Mauriello, ${ }^{2}$ and Enrico Finazzi Agrò ${ }^{1}$ \\ ${ }^{1}$ Department of Urology, Policlinico Tor Vergata, Rome, Italy \\ ${ }^{2}$ Anatomic Pathology, Department of Experimental Medicine and Surgery, Tor Vergata University, Rome, Italy
}

Correspondence should be addressed to Daniele Bianchi; danielebianchimail@yahoo.it

Received 16 April 2017; Revised 2 July 2017; Accepted 20 July 2017; Published 22 August 2017

Academic Editor: Marcus L. Quek

Copyright (C) 2017 Daniele Bianchi et al. This is an open access article distributed under the Creative Commons Attribution License, which permits unrestricted use, distribution, and reproduction in any medium, provided the original work is properly cited.

IgG4-related disease (IgG4-RD) is a nosological entity defined as a chronic immune-mediated fibro-inflammatory condition characterized by a tendency to form tumefactive, tissue-destructive lesions or by organ failure. Urologic involvement in IgG4-RD has been described in some short series of patients and in isolated case reports, most often involving the kidneys in so-called IgG4related kidney disease (IgG4-RKD). The disease can occasionally mimic malignancies and is at risk of being misdiagnosed due to its rarity. We report the case of a 56-year-old man presenting with a right renal mass suspected of being malignant. Laboratory tests showed normal creatinine levels, a high erythrocyte sedimentation rate, and high levels of C-reactive protein and microalbuminuria. The patient underwent radical right nephroureterectomy and histopathologic examination revealed features proving IgG4-RKD. He was therefore referred to immunologists. Typical clinical presentation of IgG4-RKD includes altered renal function with inconstant or no radiologic findings. Conversely, in the case we presented, a single nodule was detected upon imaging evaluation, thus mimicking malignancy. This raises the issue of a proper differential diagnosis. A multidisciplinary approach can be useful, although in clinical practice the selection of patients suspected of having IgG4-RKD is critical in the cases presenting with a renal mass that mimics malignancy.

\section{Introduction}

IgG4-related disease (IgG4-RD) is a recent nosological entity defined as a chronic immune-mediated fibro-inflammatory condition [1] characterized by tumefactive, tissue-destructive lesions or by organ failure [2]. IgG4-RD potentially involves nearly every anatomic site $[3,4]$, occasionally including urologic structures, as described in some short series of patients and in isolated case reports [5]. Typical histopathologic features are lymphoplasmacytic infiltrate rich in IgG4 plasma cells, obliterative phlebitis, and storiform fibrosis, while laboratory tests may reveal an inconstantly elevated serum IgG4 concentration [6].

IgG4-related kidney disease (IgG4-RKD) is the most common among urologic manifestations of IgG4-RD, usually presenting in the form of tubulointerstitial nephritis
(TIN) [7-9], although some cases of membranous glomerulonephritis (MGN) have been described [9-11]. IgG4-RKD presenting as a solid renal mass has rarely been described [5], and conventional imaging-including ultrasound scans, computed tomography (CT), and magnetic resonance imaging (MRI) - has proven to be of limited usefulness in determining IgG4-RD [12].

\section{Case Report}

We report the case of a 56-year-old Caucasian man presenting with a right renal mass $(12 \times 9 \times 8 \mathrm{~cm})$ revealed by CT (Figure 1) and MRI (Figure 2). The mass was localized at the upper pole of the right kidney, extending to the renal fascia towards the caval vein and iliac vessels and involving the vascular 


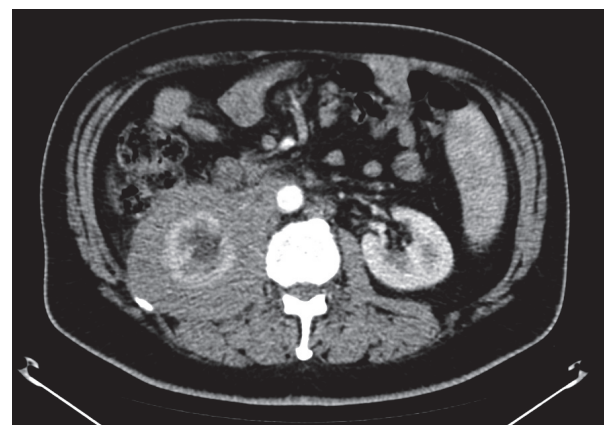

FIGURE 1: Preoperative computed tomography (CT) scan showing a right renal mass.

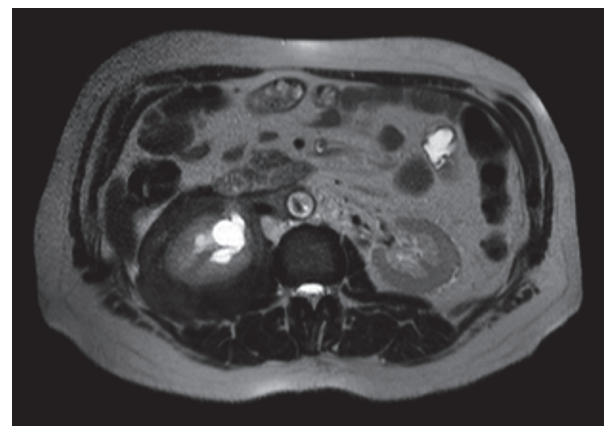

FIGURE 2: Preoperative magnetic resonance imaging (MRI) scan showing a right renal mass.

hilum. The patient complained of moderate abdominal pain over the last weeks prior to urologic consultation.

Laboratory tests performed within four months prior to urologic evaluation showed creatinine level of $1.10 \mathrm{mg} / \mathrm{dL}$, a erythrocyte sedimentation rate up to $120 \mathrm{~mm} / \mathrm{h}, \mathrm{C}$-reactive protein levels ranging from 65 to $70 \mathrm{mg} / \mathrm{L}$, and microalbuminuria levels of $82.5 \mathrm{mg} / \mathrm{g}$. These high levels drove the decision for abdominal imaging. Comorbidities included mellitus diabetes type 2 . No further relevant aspects were recorded in the patient's previous history.

Due to the suspicion of malignancy, the patient underwent open-surgery radical right nephroureterectomy, which was complicated by firm adhesions between the kidney, its fascia, and surrounding structures so that the posterior renal fascia could not be excised. The postoperative period was uneventful.

Histopathologic examination showed storiform fibrosis with lymphoplasmacytic infiltrate made up of $\mathrm{B}$ and $\mathrm{T}$ cells $(\mathrm{CD} 20+, \mathrm{CD} 3+)$, as well as focal obliterative phlebitis. The IgG4+/IgG ratio was 70\% (Figure 3). Given the histologic features proving $\operatorname{IgG} 4-\mathrm{RKD}$, the patient was referred to immunologists and underwent blood tests, including total IgG $(1150 \mathrm{mg} / \mathrm{dL})$ and IgG4 $(69 \mathrm{mg} / \mathrm{dL})$. Further rheumatologic tests (rheumatoid factor, anticardiolipin antibody, cANCA, pANCA, anti-Jo-1 antibody, complement factors C3 and C4, a Waaler Rose test, and Sm-RNP) were negative.

The patient was prescribed $5 \mathrm{mg}$ prednisone daily and $50 \mathrm{mg}$ azathioprine twice daily for the first five months after surgery and experienced dramatic pain improvement. Afterwards, a drug maintenance regimen of $50 \mathrm{mg}$ azathioprine twice daily was prescribed by immunologists. Currently, at 18 months after surgery, the patient is healthy. His creatinine level is $1.8 \mathrm{mg} / \mathrm{dL}$, his erythrocyte sedimentation rate is $17 \mathrm{~mm} / \mathrm{h}$, and his C-reactive protein level is $7.71 \mathrm{mg} / \mathrm{L}$.

\section{Discussion}

IgG4-related disease (IgG4-RD) is a recent nosological entity defined as a chronic immune-mediated fibro-inflammatory condition [1] characterized by tumefactive, tissue-destructive lesions or by organ failure [2]. IgG4-RD potentially involves nearly every anatomic site $[3,4]$, occasionally including urologic structures, as has been described in some short series of patients and in isolated case reports [5]. Typical histopathologic features are lymphoplasmacytic infiltrate rich in IgG4 plasma cells, obliterative phlebitis, and storiform fibrosis, while laboratory tests may reveal an inconstantly elevated serum IgG4 concentration [6].

In 1995, the concept of autoimmune pancreatitis (AIP) was proposed by Yoshida et al. [14]; in 2001, AIP type I was associated with high IgG4 serum levels [15]; and in 2003, IgG4-RD was described as the basic systemic condition responsible for AIP type I and for extrapancreatic lesions [16]. Since then, extrapancreatic manifestations of the disease have been increasingly reported in the literature $[1,4,17]$. 


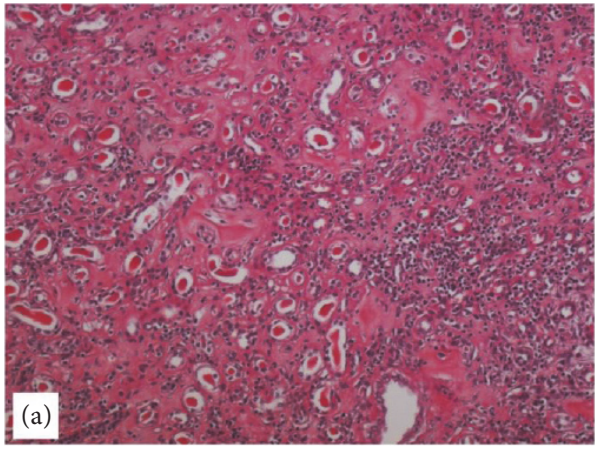

(a)

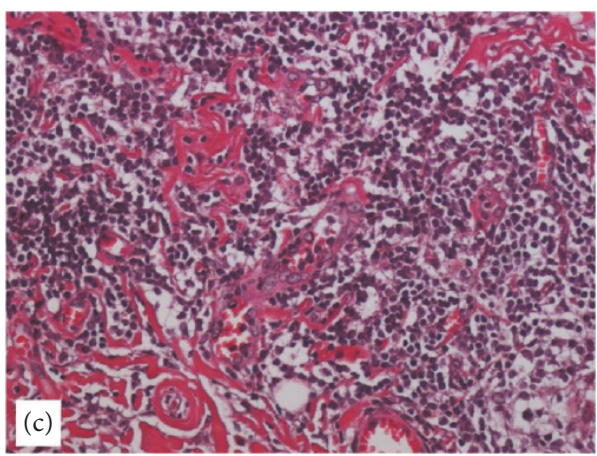

(c)

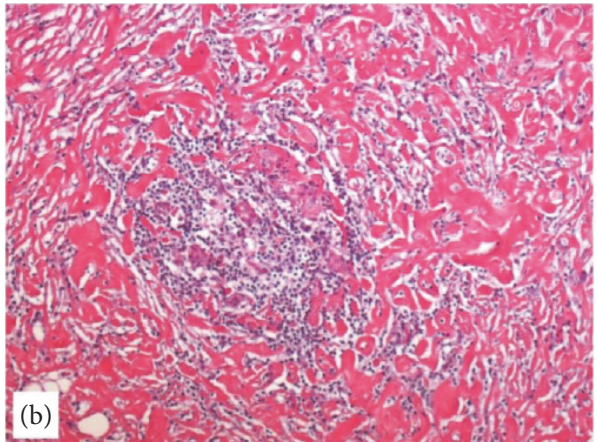

(b)

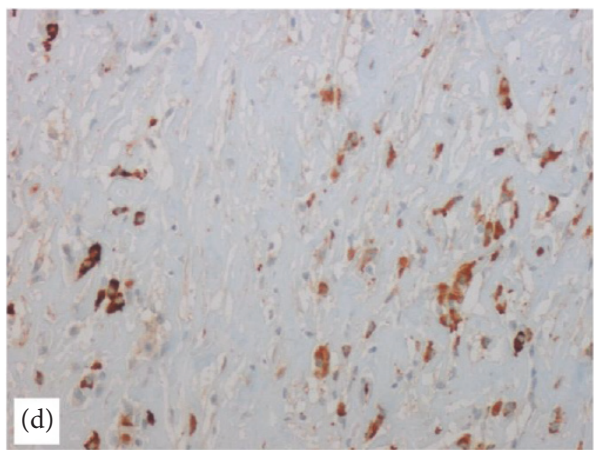

(d)

FIGURE 3: Histological findings. (a) Histological section of kidney parenchyma showing a chronic pyelonephritis characterized by the presence of interstitial fibrosis, inflammatory infiltrate of lymphocytes and plasma cells, and atrophy and dilatation of tubules that contain pink casts (hematoxylin-eosin (H-E), 10x). (b) The fibroadipose tissue of the kidney capsule typically shows the histological findings of IgG4-related disease, characterized by an irregularly whorled pattern of fibrosis (storiform fibrosis) associated with inflammatory infiltrate (H-E, 10x). (c) A vein obliterated by a transmural inflammatory cell infiltration (obliterative phlebitis) (H-E, 20x). (d) Immunostaining with anti-IgG4 antibody shows the presence of numerous positive cells $(\times 20)$.

As different terms have been proposed, the two independent teams headed by Umehara and Okazaki agreed to define the disease as IgG4-RD [18]. The disease can be difficult to diagnose because of a lack of confidence on the part of clinicians, pathologists, and radiologists [3] and because of its varying presentation [19].

Many papers have investigated urologic involvement [5], with the kidney being the most studied organ [5]. The first case of IgG4-RKD was described in 2004 [20, 21]. Serum IgG4 levels above $135 \mathrm{mg} / \mathrm{dL}$ [18] represent an important flag in the preliminary evaluation but do not represent either a necessary or sufficient condition for diagnosis [2]. However, high IgG4 serum levels seem to be related to the disease extension [2].

Our patient presented with normal IgG4 serum levels, sampled one month after surgery.

However, his IgG4/IgG ratio upon histopathologic examination was $>40 \%$, which is considered a diagnostic criterion of IgG4-RKD [6].

IgG4-RKD is the most common among urologic manifestations of IgG4-RD, usually presenting in the form of TIN [7-9], although some cases of MGN have been described [911]. The diagnostic algorithm proposed by Kawano et al. [13] is shown in Table 1, and the algorithm by Raissian et al. [10] is summarized in Table 2.

In 2011, Kawano et al. described a series of 41 patients identified between 2004 and 2011 in Japanese hospitals presenting with histopathologic findings consistent with IgG4RKD [13]. CT was performed for 29 patients; the most common radiologic findings were multiple low-density lesions, with other less frequent signs being diffuse bilateral renal swelling and diffuse thickening of the renal pelvis. A solitary hypovascular parenchymal nodule was detected in just one patient in this study; another patient probably had a unilateral renal mass causing renal swelling, but contrast-enhanced CT was not feasible because of decreased renal function. Of interest in the case we report, IgG4-RKD presented as a solid mass, thus mimicking malignancy. This is important because a differential diagnosis between IgG4-RKD and a renal neoplastic lesion is challenging.

It has been reported that conventional imagingincluding ultrasound scans, $\mathrm{CT}$, and MRI-has proven to be of limited usefulness in determining IgG4-RD [12].

Over the past decades, the management of overall renal masses has evolved from radical surgery to minimally invasive approaches, or even active surveillance in selected 
TABLE 1: Diagnostic criteria for IgG4-related kidney disease (IgG4-RKD) proposed by Kawano et al. [13].

(1) Presence of some kidney damage, as manifested by abnormal urinalysis or urine marker(s) or decreased kidney function with either elevated serum IgG level, hypocomplementemia, or elevated serum IgE level

(2) Abnormal renal radiologic findings:

(a) Multiple low-density lesions on enhanced computed tomography

(b) Diffuse kidney enlargement

(c) Hypovascular solitary mass in the kidney

(d) Hypertrophic lesion of renal pelvic wall without irregularity of the renal pelvic surface

(3) Elevated serum IgG4 level (IgG4 $\geq 135 \mathrm{mg} / \mathrm{dl}$ )

(4) Histologic findings in the kidney

(a) Dense lymphoplasmacytic infiltration with infiltrating IgG4-positive plasma cells > 10/high-power field (HPF) and/or

IgG4/IgG-positive plasma cells > 40\%

(b) Characteristics fibrosis surrounding nests of lymphocytes and/or plasma cells

(5) Histologic findings in extrarenal organ(s):

Dense lymphoplasmacytic infiltration with infiltrating IgG4-positive plasma cells > 10/HPF and/or IgG4/IgG-positive plasma cells > 40\% in extrarenal organ(s)

Definite:

$$
\begin{aligned}
& (1)+(3)+(4)(a),(b) \\
& (2)+(3)+(4)(a),(b) \\
& (2)+(3)+(5) \\
& (1)+(3)+(4)(a)+(5)
\end{aligned}
$$

Probable:

$$
\begin{aligned}
& (1)+(4)(a),(b) \\
& (2)+(4)(a),(b) \\
& (2)+(5) \\
& (3)+(4)(a),(b)
\end{aligned}
$$

Possible:
$(1)+(3)$
(2) $+(3)$
$(1)+(4)(a)$
$(2)+(4)(a)$

Appendix:

(1) Clinically and histologically, the following diseases should be excluded: Wegener's granulomatosis, Churg-Strauss syndrome, and extramedullary plasmacytoma

(2) Radiologically, the following diseases should be excluded: malignant lymphoma, urinary tract carcinomas, renal infarction, and pyelonephritis (rarely, Wegener's granulomatosis, sarcoidosis, and metastatic carcinoma)

(3) Cases with suspected disease according to the diagnostic algorithm are classified into probable or possible IgG4-RKD according to these criteria

cases. Thus, the use of renal biopsy has shown an increase, although with a lack of standardization. Consequently, the identification of patients who may benefit from renal biopsy still remains an individualized clinical decision [22]. Nevertheless, renal biopsy represents a mainstay in the diagnosis of IgG4-RKD, including the differentiation between TIN and MGN [23, 24].

In our case, prior knowledge of the proper diagnosis could have directed the management towards medical therapy, thereby avoiding surgery at least as the first option (with the proviso that a biopsy would have given specific results that ruled out malignancy). That said, a smaller renal mass would definitely have been easier to manage for both diagnosis and treatment, because retrospective analysis could not establish whether our patient might have been an adequate responder to medical therapy due to the mass volume.

\section{Conclusions}

Concerning urologic sites, kidney is the organ most frequently involved in IgG4-RD.

However, the disease is rare and can be difficult to diagnose. Its typical clinical presentation includes altered renal function, with inconstant radiologic findings. In the case presented here, a single solid nodule was detected upon imaging evaluation, thus mimicking malignancy. This raises the issue of a proper preliminary differential diagnosis, which is not always easy. Conventional imaging-including ultrasound 
TABle 2: Diagnostic criteria for IgG4-related tubulointerstitial nephritis (TIN) proposed by Raissian et al. [10].

\begin{tabular}{|c|c|}
\hline Histology & $\begin{array}{l}\text { Plasma cell-rich tubulointerstitial nephritis with } \\
>10 \text { IgG4+ plasma cells/HPF in the most } \\
\text { concentrated field }{ }^{\mathrm{a}} \\
\text { Tubular basement membrane immune complex } \\
\text { deposits by immunofluorescence, } \\
\text { immunohistochemistry, and/or electron } \\
\text { microscopy }^{\mathrm{b}}\end{array}$ \\
\hline Imaging & $\begin{array}{l}\text { Small peripheral low-attenuation cortical nodules, } \\
\text { round or wedge-shaped lesions, or diffuse patchy } \\
\text { involvement } \\
\text { Diffuse marked enlargement of kidneys }\end{array}$ \\
\hline Serology & Elevated serum IgG4 or total IgG level \\
\hline $\begin{array}{l}\text { Other organs } \\
\text { involvement }\end{array}$ & $\begin{array}{l}\text { Including autoimmune pancreatitis, sclerosing } \\
\text { cholangitis, inflammatory masses in any organ, } \\
\text { sialadenitis, inflammatory aortic aneurysm, lung } \\
\text { involvement, and retroperitoneal fibrosis }\end{array}$ \\
\hline
\end{tabular}

Diagnosis of IgG4-TIN requires the histologic feature of plasma cell-rich TIN with increased IgG4+ plasma cells and at least one other feature from the categories of "imaging," "serology," or "other organ involvement"; a mandatory criterion; ${ }^{b}$ supportive criterion, present in $>80 \%$ of cases.

scans, CT, and MRI-has proven to be of limited usefulness in determining IgG4-RD. A multidisciplinary approach can be useful, although in clinical practice the selection of patients suspected of having IgG4-RKD is critical in cases presenting as a renal mass, thus mimicking malignancy.

\section{Ethical Approval}

The research was carried out in compliance with the Helsinki Declaration: Ethical Principles for Medical Research Involving Human Subjects.

\section{Consent}

Written informed consent was obtained from the patient.

\section{Disclosure}

No biostatistical analysis was needed.

\section{Conflicts of Interest}

The authors declare that they have no conflicts of interest.

\section{References}

[1] Z. S. Wallace and J. H. Stone, "An update on IgG4-related disease," Current Opinion in Rheumatology, vol. 27, no. 1, pp. 8390, 2015.

[2] A. Khosroshahi, Z. S. Wallace, and J. L. Crowe, "International consensus guidance statement on the management and treatment of IgG4-related disease," Arthritis \& Rheumatology, vol. 67, no. 7, pp. 1688-1699, 2015.
[3] M. N. Carruthers, J. H. Stone, and A. Khosroshahi, "The latest on IgG4-RD," Current Opinion in Rheumatology, vol. 24, no. 1, pp. 60-69, 2012.

[4] H. Umehara, K. Okazaki, Y. Masaki et al., "A novel clinical entity, IgG4-relateddisease (IgG4RD): general concept and details," Modern Rheumatology, vol. 22, no. 1, pp. 1-14, 2012.

[5] D. Bianchi, "IgG4-related disease: what urologists should know," International Urology and Nephrology, vol. 48, no. 3, pp. 301-312, 2016.

[6] V. Deshpande, Y. Zen, J. K. Chan et al., "Consensus statement on the pathology of IgG4-related disease," Modern Pathology, vol. 25, no. 9, pp. 1181-1192, 2012.

[7] L. D. Cornell, S. L. Chicano, V. Deshpande et al., "Pseudotumors due to IgG4 immune-complex tubulointerstitial nephritis associated with autoimmune pancreatocentric disease," The American Journal of Surgical Pathology, vol. 31, no. 10, pp. 15861597, 2007.

[8] T. Saeki, S. Nishi, N. Imai et al., "Clinicopathological characteristics of patients with IgG4-related tubulointerstitial nephritis," Kidney International, vol. 78, no. 10, pp. 1016-1023, 2010.

[9] S. J. Watson, D. A. Jenkins, and C. O. Bellamy, "Nephropathy in IgG4-related systemic disease," The American Journal of Surgical Pathology, vol. 30, no. 11, pp. 1472-1477, 2006.

[10] Y. Raissian, S. H. Nasr, and C. P. Larsen, "Diagnosis of IgG4related tubulointerstitial nephritis," Journal of the American Society of Nephrology, vol. 22, no. 7, pp. 1343-1352, 2011.

[11] J. Morimoto, Y. Hasegawa, H. Fukushima et al., "Membranoproliferative glomerulonephritis-like glomerular disease and concurrent tubulointerstitial nephritis complicating IgG4-related autoimmune pancreatitis," Internal Medicine, vol. 48, no. 3, pp. 157-162, 2009.

[12] M. Horger, H. Lamprecht, R. Bares et al., "Systemic IgG4related sclerosing disease: spectrum of imaging findings and differential diagnosis," American Journal of Roentgenology, vol. 199, no. 3, pp. W276-W282, 2012.

[13] M. Kawano, T. Saeki, H. Nakashima et al., "Proposal for diagnostic criteria for IgG4-related kidney disease," Clinical and Experimental Nephrology, vol. 15, no. 5, pp. 615-626, 2011.

[14] K. Yoshida, F. Toki, T. Takeuchi, S.-I. Watanabe, K. Shiratori, and N. Hayashi, "Chronic pancreatitis caused by an autoimmune abnormality. Proposal of the concept of autoimmune pancreatitis," Digestive Diseases and Sciences, vol. 40, no. 7, pp. 1561-1568, 1995.

[15] H. Hamano, S. Kawa, A. Horiuchi et al., "High serum IgG4 concentrations in patients with sclerosing pancreatitis," The New England Journal of Medicine, vol. 344, no. 10, pp. 732-738, 2001.

[16] T. Kamisawa, N. Funata, Y. Hayashi et al., "A new clinicopathological entity of IgG4-related autoimmune disease," Journal of Gastroenterology, vol. 38, no. 10, pp. 982-984, 2003.

[17] M. Guma and G. S. Firestein, "IgG4-related diseases", Best Practice and Research: Clinical Rheumatology, vol. 26, no. 4, pp. 425-438, 2012.

[18] H. Umehara, K. Okazaki, Y. Masaki et al., "Comprehensive diagnostic criteria for IgG4-related disease (IgG4-RD), 2011," Modern Rheumatology, vol. 22, no. 1, pp. 21-30, 2012.

[19] N. Seo, J. H. Kim, J. H. Byun, S. S. Lee, H. J. Kim, and M.-G. Lee, "Immunoglobulin G4-related kidney disease: A comprehensive pictorial review of the imaging spectrum, mimickers, and clinicopathological characteristics," Korean Journal of Radiology, vol. 16, no. 5, pp. 1056-1067, 2015. 
[20] Y. Uchiyama-Tanaka, Y. Mori, T. Kimura et al., "Acute tubulointerstitial nephritis associated with autoimmune-related pancreatitis," American Journal of Kidney Diseases, vol. 43, no. 3, pp. e18-25, 2004.

[21] S.-I. Takeda, J. Haratake, T. Kasai, C. Takaeda, and E. Takazakura, "IgG4-associated idiopathic tubulointerstitial nephritis complicating autoimmune pancreatitis," Nephrology Dialysis Transplantation, vol. 19, no. 2, pp. 474-476, 2004.

[22] M. Haifler and A. Kutikov, "Current Role of Renal Biopsy in Urologic Practice," Urologic Clinics of North America, vol. 44, no. 2, pp. 203-211, 2017.

[23] H. J. Jeong, S. Shin, and B. J. Lim, "Overview of IgG4Related Tubulointerstitial Nephritis and Its Mimickers," Journal of Pathology and Translational Medicine, vol. 50, no. 1, pp. 2636, 2016.

[24] H. Nakashima, M. Kawano, T. Saeki et al., "Estimation of the number of histological diagnosis for IgG4-related kidney disease referred to the data obtained from the Japan Renal Biopsy Registry (J-RBR) questionnaire and cases reported in the Japanese Society of Nephrology Meetings," Clinical and Experimental Nephrology, vol. 21, no. 1, pp. 97-103, 2017. 


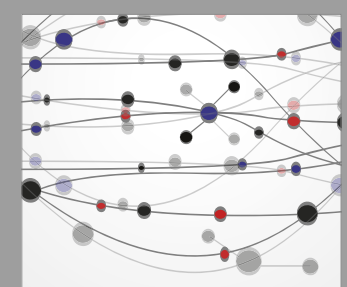

The Scientific World Journal
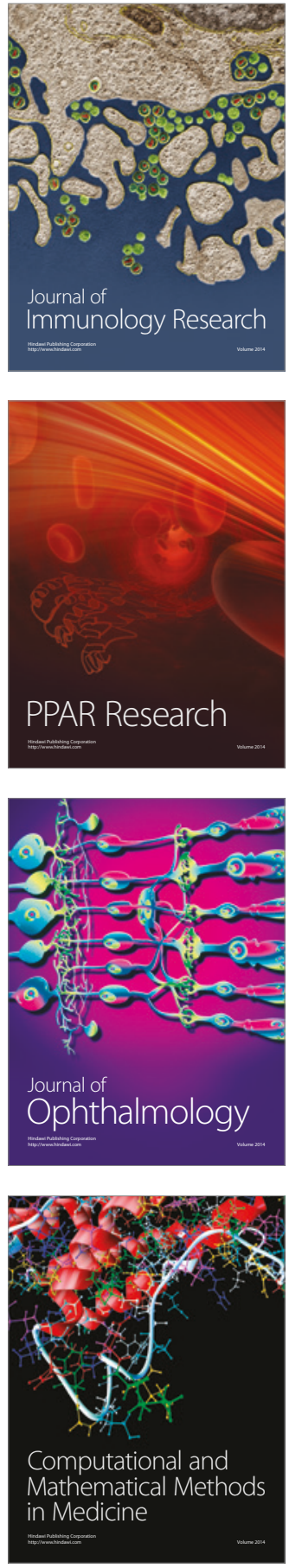

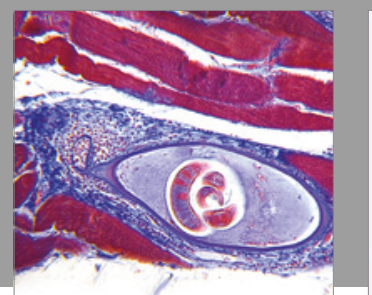

Gastroenterology Research and Practice
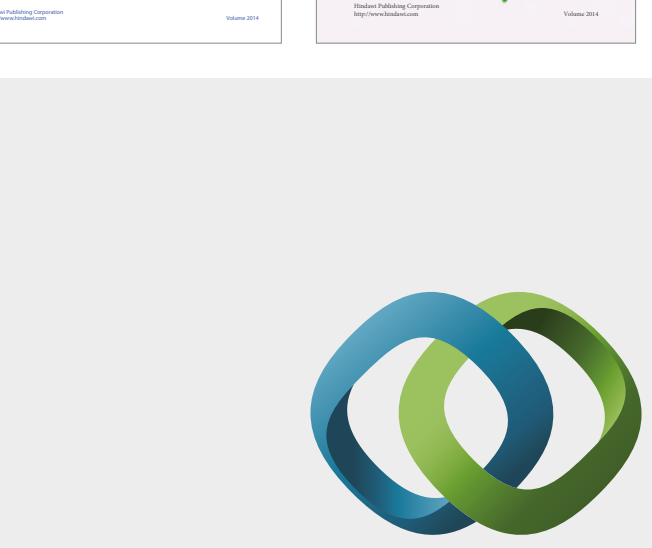

\section{Hindawi}

Submit your manuscripts at

https://www.hindawi.com
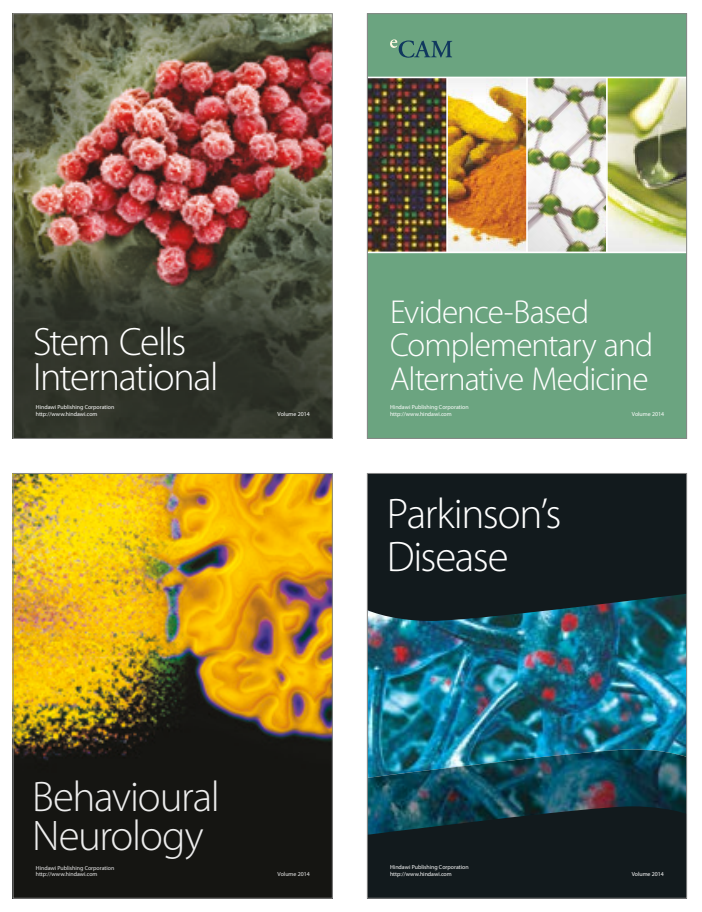
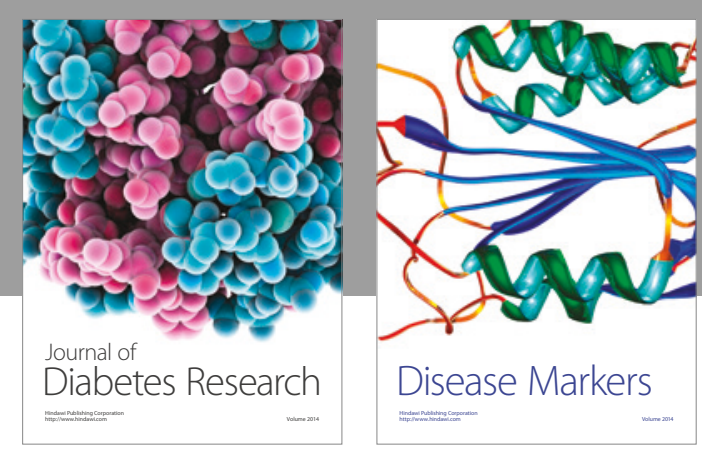

Disease Markers
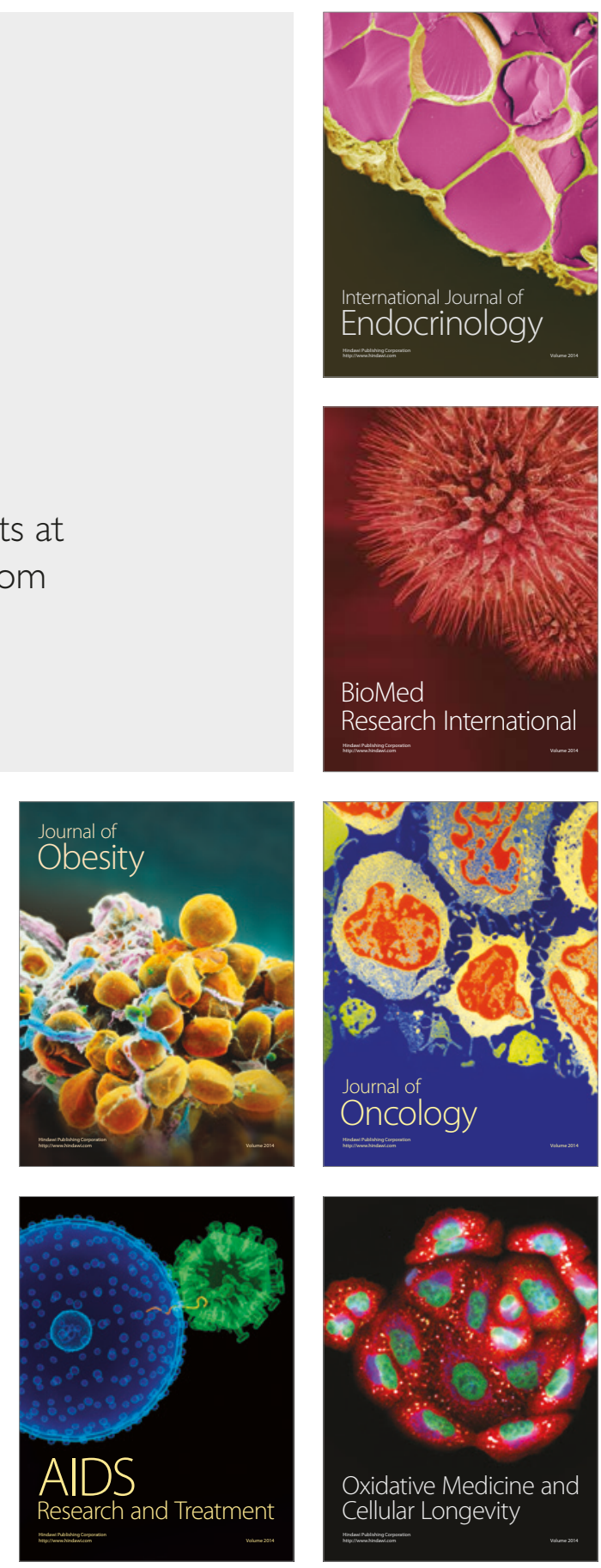